\title{
Pacientes com transtornos mentais que buscam atendimento em centro de saúde psicossocial no Brasil- um estudo de revisão
}

\section{Patients with mental disorders who seek for attention in psychosocial health center in Brazil - a review study}

Isabel Cristina Borges Esmério1, Rayssa Claudia Oliveira Duarte ${ }^{1}$, Breno Soares Borges Oliveira1, Wemerson Davi Miranda1, Eduardo Fernandes de Miranda², Laís Tonello ${ }^{3}$

\section{RESUMO}

Introdução: Os transtornos mentais são denominados como alterações de humor ou modo de pensar, os quais impactam negativamente no desempenho pessoal em diversos âmbitos. A depressão e os transtornos de humor são os mais recorrentes. Os Centro de Atenção Psicossocial (CAPS) oferecem tratamentos diversos à esses pacientes, visando reabilitação e reintegração social. Objetivo: Traçar o perfil epidemiológico de pessoas que buscam atendimento em CAPS. Metodologia: Estudo descritivo a partir de buscas na Biblioteca Virtual de Saúde. Foram incluídos sete artigos científicos que abordam sobre o perfil das pessoas que buscam tratamento nos CAPS. Resultados: A faixa etária mais frequente está entre 40 e 69 anos; a profissão mais recorrente é "do lar". A renda familiar, quando analisada, varia entre 1 a 3 salários mínimos. O estado civil prevalente é solteiro. Os transtornos mais frequentes foram esquizofrenia, transtornos esquizotípicos e transtornos delirantes (F20-29). Conclusão: a partir dos dados analisados, pode-se de dizer que, mulheres adultas/idosas, solteiras, de baixa condição socioeconômica, e com diagnóstico médico de esquizofrenia, são maioria dos pacientes atendidos pelos CAPS.

Palavras-chave: Perfil Epidemiológico. Impacto Psicossocial. Saúde mental.

\section{ABSTRACT}

Introduction: Mental disorders are called mood or thinking disorders, which have a negative impact on personal performance in various settings. Depression and mood disorders are the most recurrent. The Psychosocial Care Center (CAPS) offers different treatments to these patients, aiming at rehabilitation and social reintegration. Objective: To define the epidemiological profile of people seeking care in CAPS. Methodology: A descriptive study based on searches in the Virtual Health Library. Seven scientific articles were included that address the profile of people seeking treatment in CAPS. Results: The most frequent age group is between 40 and 69 years old; the most recurring profession is "home". Family income, when analyzed, ranges from 1 to 3 minimum wages. The prevailing marital status is single. The most frequent disorders were schizophrenia, schizotypal disorders and delusional disorders (F20-29). Conclusion: from the analyzed data, it can be said that adult / single women, single, with low socioeconomic status, and with a medical diagnosis of schizophrenia, are the majority of the patients served by CAPS.

Keywords: Epidemiological profile. Psychosocial Impact. Mental health 


\section{INTRODUÇAOOO}

O termo "transtornos mentais" é usado para categorizar uma diversidade de transtornos mentais e comportamentais os quais são abordados pela Classificação Internacional de Transtornos Mentais e de Comportamento (CID-10), incluindo distúrbios causadores de uma variedade de doenças, tais como depressão, transtorno afetivo bipolar, esquizofrenia, transtornos de ansiedade, transtornos por uso de substância, esquizofrenia e demais. Inclui-se como determinantes de saúde mental e dos transtornos mentais os atributos indivíduos, como a capacidade de administrar pensamentos, emoções, comportamentos, interações sociais, além dos fatores sociais, políticos, culturais e econômicos. Apesar de existir indivíduos e grupos da sociedade com risco mais proeminente de sofrer problemas de saúde mental, ainda são considerados universais, atingem qualquer faixa etária, e também causa incapacitações graves e definitivas. São universais e atinge indivíduos de qualquer faixa etária, e tem causado incapacitações graves e definitivas (WHO, 2013).

Um estudo da Organização Mundial da Saúde, realizado no ano de 2000, demonstrou uma estimativa de $36 \%$ das pessoas no Brasil irão desenvolver algum tipo de transtorno mental durante suas vidas (FIOROTTI; TOMAZELLI; MALAGRIS, 2009). Já em 2011, o Ministério da Previdência Social, apontou os transtornos mentais e comportamentais como a terceira maior causa das concessões de auxílio-doença por incapacidade laboral (CARLOTTO, 2017).

Para atender essa demanda, o Centro de Atenção Psicossocial (CAPS), é um serviço de atendimento em saúde mental criado para substituir os hospitais psiquiátricos e oferecem tratamentos diversos à pacientes de transtornos mentais visando reabilitar e reintegrar à vida social (BRASIL, 2015). Apesar que, em 2014 já existiam cerca de 2209 unidades distribuídas em todo território nacional, e a crescente quantidade de estruturas sendo instaladas, ainda é insuficiente para atender as reais necessidades, enquadrando a saúde mental como um problema de saúde pública no país (BRASIL, 2015).

Colocar em prática essa política nacional, confronta com questões socioeconômicas e limitantes da própria doença, por exemplo, apesar de ter o serviço especializado, a falta de informação dos familiares e a má adesão dos pacientes psiquiátricos ao tratamento farmacológico, dificultam a terapia (CARDOSO e FRARI GALERA, 2009). Outrora, o encaminhamento errôneo de pacientes acarreta problemas estruturais e falta de mão de obra especializada, um exemplo é o caso de pacientes dependentes químicos serem 
atendidos em unidades de atendimento não especifica, gerando desorganização na prestação de assistência de saúde mental dos seus usuários (PEREIRA, 2012).

Dessa forma, se torna importante conhecer melhor os pacientes atendidos nos CAPS, pois os estudos epidemiológicos no campo da psiquiatria são considerados mais complexos porque os processos psíquicos sofrem influências de fatores sociais, econômicos e culturais (OLIVEIRA, et al., 2015). Os estudos apresentados na literatura ainda não são claros em relação ao perfil dos pacientes que buscam os tratamentos nos CAPS pelo Brasil, pois apresentam informações apenas de forma localizada, cidade onde foi realizada a pesquisa, e nenhum estudo apresenta informações de uma forma ampliada de uma região ou um perfil nacional.

Desta forma, este estudo busca apresentar, a partir da literatura publicada, qual o perfil das pessoas que buscam o atendimento nos CAPS no território nacional. Sendo assim o objetivo do presente estudo foi traçar o perfil epidemiológico de pessoas que buscam atendimento em Centros de Atenção Psicossocial (CAPS), a partir de uma revisão da literatura na Biblioteca Virtual em Saúde.

\section{MATERIAIS E METODOS}

Foi realizada uma busca relevante de estudos científicos na biblioteca virtual em saúde (BVS). Utilizou-se como palavras chave para busca: perfil; psicossocial e saúde mental, elas poderiam estar vinculadas ao título, resumo ou assunto. $O$ único filtro adotado foi ter o texto completo disponível. Os resultados dessa pesquisa totalizaram 147 trabalhos, sendo artigos científicos, dissertações, teses, e resumos expandidos. Utilizou-se como critérios de inclusão: artigos científicos, escritos em português, no qual a principal temática do estudo estivesse relacionada com o perfil das pessoas que procuraram atendimentos de serviço de saúde no âmbito de saúde mental e que não fossem restritos a uma determinada faixa etária ou grupo social. Ao todo, sete artigos atenderam aos critérios estabelecidos e foram selecionados. A seleção dos artigos foi realizada em maio de 2017, por dois pesquisadores de forma independente.

\section{RESULTADOS}

Foram incluídos os resultados de sete artigos que abordavam avaliações do perfil dos pacientes que procuram atendimentos nos CAPS do Brasil. Na tabela 1 são apresentadas a cidade onde a pesquisa foi realizada, como a quantidade de sujeitos, a idade, e o CID-10 que caracterizou a doença destes pacientes. 
Tabela 1 - Características dos estudos revisados

\begin{tabular}{ccccc}
\hline AUTORES/ANO & CIDADE/ESTADO & SUJEITOS & $\begin{array}{c}\text { IDAD } \\
\text { E }\end{array}$ & CID-101 \\
\hline Pelisoli e Moreira (2005) & Osório/RS & 2470 & $30-35$ & $\begin{array}{c}\text { F30-39; F40-48 } \\
\text { F90-98 }\end{array}$ \\
\hline Carvalho et al. (2010) & Iguatu/CE & $\begin{array}{c}\text { CAPS geral 58 } \\
\text { CAPS AD 47 } \\
\text { CAPSi 38 }\end{array}$ & $\begin{array}{c}42 \\
41,9 \\
10,8\end{array}$ & $\begin{array}{c}\text { F10 } \\
\text { F40-48 }\end{array}$ \\
\hline Freitas e Souza (2010) & Ilhéus/BA & 223 & 43 & F20; F32; F29 \\
\hline Pereira et al. (2012) & Lorena/SP & 5490 & $40-69$ & F40-48; F10-19 \\
\hline Mangualde et al. (2013) & Barbacena/MG & 583 & $31-40$ & F20-29; F30-48 \\
\hline Reis et al. (2013) & Ribeirão Preto/ SP & 244 & $40-49$ & F60-69 \\
\hline Cruz et al. (2016) & Candeias/BA & 71 & $30-39$ & F20-29 \\
\hline
\end{tabular}

${ }^{1}$ CID-10: Classificação Estatística Internacional de Doenças e Problemas Relacionados com a Saúde; F10: Transtornos comportamentais por abuso de álcool; F10-F19: Transtornos mentais e comportamentais em razão do uso de substâncias psicoativas; F20: Esquizofrenia; F20-29: Esquizofrenia, transtornos esquizotípicos e transtornos delirantes; F29: Psicose não orgânica não especificada; F30-39: Transtornos de humor; F32: Episódios depressivos; F30-48: Transtornos de Humor e Transtornos neuróticos relacionados ao stress e transtornos somatoformes F40-48: Transtornos neuróticos relacionados ao stress e transtornos somatoformes; F60-69: Transtorno de personalidade; F90-98: Transtornos com início na infância e adolescência.

Na tabela 2 são apresentados o perfil dos pacientes atendidos em CAPS, segundo os artigos selecionados para este estudo.

\section{DISCUSSAOO}

Observou-se que Freitas e Souza (2010), Pereira et al. (2012), Reis et al., (2013) Carvalho et al., (2010) mostram que a faixa etária mais frequente nos CAPS é entre 40 e 69 anos, com 223, 5490, 244 e 105 sujeitos em suas amostras, respectivamente. Nem todos os artigos estudados analisaram a profissão dos sujeitos, mas pode-se observar que dois artigos mostram a profissão "do lar" a mais recorrente entre os pacientes (FREITAS E SOUZA, 2010; PEREIRA et al., 2012).

No que se refere ao sexo, quatro artigos obtiveram a prevalência do sexo feminino e em outros três a prevalência do sexo masculino dentre os pacientes dos CAPS. PEREIRA et al., (2012) traz uma prevalência feminina no Ambulatório de Saúde Mental (ASM) (67,6\%) e masculina no CAPS (61,4\%). Pelisoli e Moreira (2005) comparam os sexos e os transtornos, sendo o sexo masculino o maior número de diagnósticos de transtornos, com início na infância e adolescência (30,98\%) e o sexo feminino, com transtornos de humor (27,92\%). Em Carvalho et al. (2010), o sexo masculino é a maioria dos atendidos no CAPS geral, CAPSad (álcool e drogas) e CAPSi (infantil). 
Tabela 2 - Perfil dos pacientes dos artigos selecionados

\begin{tabular}{|c|c|}
\hline Autores & Objetivo \\
\hline $\begin{array}{l}\text { Pelisoli e } \\
\text { Moreira, } \\
\text { (2005) }\end{array}$ & $\begin{array}{c}\text { Delinear o perfil dos } \\
\text { usuários da } \\
\text { instituicão }\end{array}$ \\
\hline
\end{tabular}

prontuários

Sexo: $59,6 \%$ - ${ }^{*}$ F. Média de idade: 30,35 anos. Ocupação: estudante $(27,4 \%)$, do lar $(13 \%)$, doméstica $(2,5 \%)$, aposentado $(1,9 \%)$ e agricultor $(1,7 \%)$. Média de atendimentos por paciente $=6,77$. Situação do tratamento: não-aderência (54\%). Presença de al guma internação psiquiátrica: $5,9 \%$. Categoria diagnóstica prevalente: Transtornos com início na infância e adolescência - 21,69\%. Hipótese diagnóstica mais relevante estatisticamente: Episódio depressivo (F32) - 10,5\%. Categorias diagnósticas prevalentes por sexo: *F-Transtornos do humor, 27,92\% e Transtornos de ansiedade, 27,42\%; ${ }^{* *} \mathrm{M}$ : Transtornos com início na infância e adolescência - 30,98\% e Esquizofrenia, transtorno esquizotípico e delirante $-11,90 \%$. Profissionais consultados: Psiquiatria-39\%, Psicologia - 37\%. Origem da demanda: espontânea (70,2\%).

Média de idade: ${ }^{* \star *}$ CAPS Geral $=42$ anos. ${ }^{+}$CAPS AD $=41,9$ anos: ${ }^{++}$CAPSi $=10,8$ anos. Sexo: ${ }^{* *}$ M: CAPS AD (85,1\%), CAPSi $(71,1 \%)$, ${ }^{*}$ F CAPS Geral (79,3\%); Escolaridade predominante: 4 a 7 anos: ${ }^{+}$CAPS AD- $42,6 \%$, ${ }^{++}$CAPSi- 36,7\%, CAPS Geral- 39,7\%; Renda familiar: 1 a 2 Saláriosmínimo: CAPS AD $(51,1 \%)$, Não especificado- CAPSi: $100 \%$, CAPS Geral:79,3\%. Estado civil: Solteiro: CAPS AD- 59,6\%, CAPSi-100\%,

Descrever o perfil epidemiológico dos usuários assistidos

Carvalho,et al. (2010) Atenção

Psicossocial (CAPS) do município

Casado: CAPS Geral- 51,7\%; Origem do encaminhamento ao CAPS: Família: CAPS AD- 46,9\%, CAPSi- 60,5\%, CAPS Geral- 69\%;

Análise de Acompanhamento profissional: Mensal - CAPS AD- $(53,1 \%)$, CAPS Geral prontuários (37,9\%); Semanal: CAPSi $(63,2 \%)$; Medicação contínua: Sim: CAPS AD $(89,4 \%)$, CAPS Geral (100\%); Não: CAPSi- 52,6 \%. Internação psiquiátrica: Não: CAPS AD- 97,9\%, CAPSi-100\%, CAPS Geral- 63,8\%. Modalidades terapêuticas: Atendimento individual e coletivo: CAPS AD: 95,7\%, CAPSi- 50\%, Atendimento individual: CAPS Geral- $81 \%$. Diagnóstico prevalente: CAPS AD: transtornos comportamentais por abuso de álcool $(n=43)$, CAPSi: transtornos ansiosos $(n=8)$, CAPS Geral: transtornos de ansiedade $(n=17)$. 


\begin{tabular}{|c|c|c|}
\hline $\begin{array}{c}\text { Freitas e } \\
\text { Souza } \\
(2010)\end{array}$ & $\begin{array}{l}\text { Investigar o conjunto } \\
\text { de características } \\
\text { clínicas e } \\
\text { sociodemográficas } \\
\text { dos usuários do } \\
\text { Centro de Atenção } \\
\text { Psicossocial (CAPS) } \\
\text { do município de } \\
\text { Ilhéus, Bahia }\end{array}$ & $\begin{array}{c}\text { Dados } \\
\text { coletados dos } \\
\text { prontuários e } \\
\text { mediante } \\
\text { entrevista com } \\
\text { os pacientes }\end{array}$ \\
\hline
\end{tabular}

\section{Identificar o perfil dos usuários do (2012) "ASM e do ${ }^{* * *}$ CAPS I deste município}

Análise de prontuários

Descrever o perfil Mangualde et epidemiológico dos al. (2013) no ${ }^{* *}$ CAPS de Barbacena
Idade média: 43 anos. Sexo: ${ }^{* \star} \mathrm{M}(52 \%)$. Regime de atendimento: nãointensivo (45\%), semi-intensivo (36,8\%) e intensivo (18,2\%). Religião: 36,8\% evangélicos. Ocupação: $21,0 \%$ "do lar", 16,0\% aposentados ou beneficiários da Previdência Social, 13,3\% desempregados. Cuidador familiar: Outro (filho, tio, sobrinho): 35,1\%, Mãe: 29,7\%. Tipo da habitação: Alvenaria: $59,2 \%$. Procedência: $87,4 \%$ residência fora do bairro em que está implantado $0{ }^{* * *}$ CAPS. Renda familiar: 40,4\%: 1 a 3 salários mínimos. Tempo de adoecimento: $\geq 10$ anos: $54,2 \%$. Tempo de acolhimento: $\geq 4$ anos: 47,5\%. Internações em Hospitais Psiquiátricos: Sim: 46,6\%. Diagnóstico predominante: esquizofrenia (30\%). Principais medicamentos utilizados: 70,4\% - antiepilépticos e anticonvulsivantes. Sexo: ${ }^{A}$ ASM: $67,6 \%{ }^{*}$ F. ${ }^{* * *}$ CAPS: $61,4 \%{ }^{* \star}$ M. Média de idade: ${ }^{*}$ ASM: 47,9 anos; ${ }^{* \star}$ CAPS: 41 anos Diagnósticos predominantes: ${ }^{~ A S M: ~ t r a n s t o r n o s ~}$ neuróticos relacionados ao estresse e transtornos somatoformes $(16,4 \%)$. ${ }^{* * *}$ CAPS: Transtornos mentais e comportamentais em razão do uso de substâncias psicoativas $(25,8 \%)$. Ocupação: ${ }^{* A S M}$ : do lar $(47,4 \%) ;{ }^{* * *}$ CAPS: do $\operatorname{lar}(20,5 \%)$. Bairro de residência: região Oeste do município (39,6\%), outros municípios (3\%); ${ }^{* *}$ CAPS: 40,5\% - região Oeste do município; outros municípios (0,9\%). Escolaridade: ${ }^{\star *}$ CAPS: $50 \%$ - Ensino Fundamental integral ou parcial; \#ASM: não obtido. Estado civil: ${ }^{* *}$ CAPS: solteiros $(60 \%)$; \#ASM: não obtido. Etnia: ${ }^{* * *}$ CAPS: brancos (73\%); "ASM: não obtido. Medicamentos mais prescritos: ${ }^{\text {ASM: }}$ antidepressivos (45\%); ${ }^{* *} \mathrm{CAPS}$ : Antipsicóticos $(44,7 \%)$.

Sexo: $56,6 \%{ }^{* *} \mathrm{M}$. Média de idade: $38,7 \pm 3,6$ anos. Estado civil: Sem informação $(39,9 \%)$, casados/amasiados $(29,1 \%)$. Escolaridade: Sem Análise de informação (62,1\%), analfabetos ou 1 Grau incompleto (22,7\%). Ocupação: prontuários Sem informação (59,8\%), assalariados $(11,9 \%)$. Origem do referente ao encaminhamento ao ${ }^{\star \star \star}$ CAPS: Serviços de saúde $(40,1 \%)$, Família $(23,1 \%)$, primeiro Espontânea (22,7\%). Procedência: Zona rural (82,5\%). História Pregressa: atendimento no Reagudização (33,6\%), Internação Prévia (27,9\%). Diagnósticos serviço predominantes: Transtornos psicóticos $(28,8 \%)$, Transtornos de humor $(22,3 \%)$. Tratamento indicado: Não intensivo $(23,7 \%)$, Internação $(22,5 \%)$. Medicação utilizada: Mais de uma (56,9\%), Sem informação $(19,6 \%)$, Antipsicótico (7,4\%), Ansiolítico (6,7\%). 


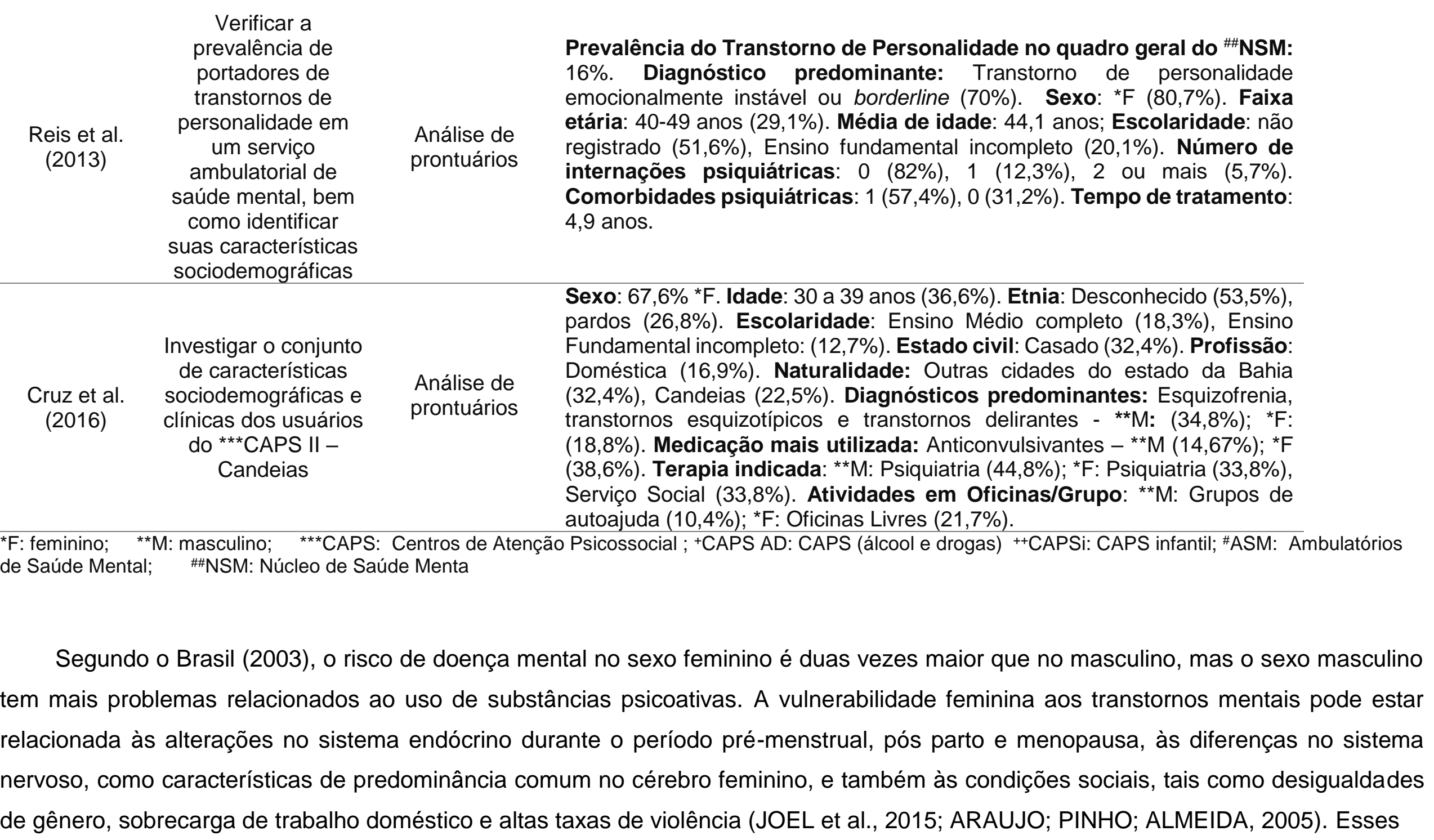


dados são sugestivos em Carvalho et al. (2010), visto que a maioria dos usuários do CAPS geral eram mulheres $(79,3 \%)$ e do CAPSad eram homens $(85,1 \%)$.

A escolaridade, em maior parte, é desconhecida por não ser pesquisada, Pelisoli e Moreira (2005) e Freitas e Souza (2010), ou porque não pôde ser colhida por falta de informação ou registro (PEREIRA et al., 2012). Além disso, 62,1\% não tem informações nos estudos de Mangualde et al. (2013), e 51,6\% e de Reis et al. (2013). Os estudos que avaliaram essa variável, podemos observar que a maioria dos sujeitos cursaram até o Ensino Fundamental incompleto ou completo (CARVALHO et al., 2010; PEREIRA et al., 2012; MANGUALDE et al., 2013; e REIS et al., 2013).

Em apenas dois artigos foi pesquisado a renda familiar, sendo que essa varia entre 1 a 2 salários mínimos (CARVALHO et al., 2010) e 1 a 3 salários mínimos (FREITAS E SOUZA, 2010). É necessário atentar que apesar de não avaliados na maioria dos artigos, a escolaridade e a renda sejam por falta de dado de informação ou por não ser objeto de estudo. Esses dados sociodemográficos são importantes para delinear a população, visto que estudos mostram que a menor renda e a baixa escolaridade são fatores de risco para o sofrimento mental (PATEL e KLEINMAN 2003).

Em relação ao estado civil, observou-se que a maioria eram solteiros, identificou que $60 \%$ dos usuários do CAPS (PEREIRA et al., 2012), 59,6\% dos usuários do CAPSad e 100\% dos usuários do CAPSi (CARVALHO, et al., 2010) eram solteiros. Já em Cruz et al. (2016) 32\% eram casados, além disso, Carvalho et al. (2010), ao analisar dados do CAPS observou que $51,7 \%$ eram casados.

Os últimos dados publicados, mostram que no Brasil, observou-se que o transtorno mental mais prevalente com diagnóstico por um profissional de saúde mental é a depressão, contando com $7,6 \%$ das pessoas de 18 anos ou mais de idade (IBGE, 2014). Já no presente estudo de revisão, notou-se que os artigos identificaram que os usuários em maior número são aqueles com diagnóstico de esquizofrenia, transtornos esquizotípicos e transtornos delirantes (F20-29) (FREITAS E SOUZA, 2010; MANDGUALDE et al., 2013; CRUZ et al., 2012). Tal divergência pode ser considerada pelo maior número de atendimentos ao transtorno depressivo ser realizado em consultório particular ou clínica privada $(42,3 \%)$ ou em unidade básica de saúde $(33,2 \%)$, sendo que somente são atendidos em CAPS 5,3\% desses pacientes (5,3\%), necessitando de estudos posteriores para elucidação (IBGE, 2014).

É importante destacar que os CAPS oferecem serviços de atendimento multiprofissional para pessoas com transtornos mentais graves e persistentes, inclusive 
pelo uso de substâncias, mas com diferenças em faixas etárias e quantitativos de habitantes na cidade. Sendo assim o CAPS I, existem em cidades com mais de 15 mil habitantes; CAPS II, em cidades com mais de 70 mil habitantes; CAPSi, atendem crianças e adolescentes em cidades ou regiões com mais de 70 mil habitantes; CAPSad, todas as faixas etárias mas especifico para pessoas com transtorno por uso de substancias em cidades com mais de 70 mil habitantes; CAPS III, oferecem até cinco vagas para atendimento noturno em todas as faixas etárias em cidades com mais de 150 mil habitantes. CAPSad III oferece entre 8 e 12 vagas de acolhimento noturno, com atendimento 24 horas, para todas as faixas etárias, específico para uso de substâncias, em cidades com pelo menos 150 mil habitantes (BRASIL, 2003).

Os impasses do trabalho consistiram em obter dados confiáveis em estudos epidemiológicos sobre o assunto, além do mais, o número de pacientes diagnosticados com transtornos mentais tem aumentado anualmente, e a falta de informação prejudica para realizar um tratamento estatístico. Há uma grande demanda de atendimentos em setores não especializados, por consequência o tempo de tratamento também se eleva (BRASIL, 2015).

É necessário ressaltar que ainda são necessários uma maior quantidade de trabalhos e esforço permanente para identificar qual o público assistido pelos CAPS, além disso, é necessário conscientizar os profissionais que trabalham com esses pacientes, para 0 preenchimento correto dos prontuários, levando em consideração dados muitas vezes menosprezados, como escolaridade, profissão, estado civil e procedência, mas importantes para determinar o perfil dos pacientes. Desta forma destinar medidas de prevenção e tratamento nessa parcela da população.

Assim, é possível mostrar aos órgãos públicos locais, a população mais propensa ao adoecimento psíquico e quais os transtornos mais frequentes para determinado perfil populacional. Dessa forma, fica fácil promover medidas mais assertivas para a adoção de estilo de vida mais saudável e distribuição mais coerente e equitativa de recursos e serviços de saúde (CAMBOTA E ROCHA, 2015).

\section{CONSIDERAÇÖES FINAIS}

Os estudos demonstraram que os pacientes atendidos nos CAPS são, em sua maioria, mulheres entre 40 a 69 anos, solteiras, de baixa renda e escolaridade, e apresentam esquizofrenia como diagnóstico médico. A determinação de um perfil 
prevalente de pessoas que buscam o atendimento do CAPS é importante para traçar estratégias e ações de políticas públicas voltadas para esse público especificamente.

De acordo com os resultados encontrados e das adversidades relatadas pelos autores, na dificuldade em obter e entender os dados, por vezes suprimidos, incompletos e ausentes, sugere-se uma discussão no âmbito nacional para padronização do preenchimento dos prontuários. Desta forma, pesquisas futuras sejam realizadas para caracterizar esse público de maneira regional, estatual e nacional, para que medidas de prevenção e tratamento sejam direcionadas a população de maior risco.

\section{REFERENCIAS}

ARAUJO, Tânia Maria de; PINHO, Paloma de Sousa; ALMEIDA, Maura Maria Guimarães de. Prevalência de transtornos mentais comuns em mulheres e sua relação com as características sociodemográficas e o trabalho doméstico. Rev. Bras. Saude Mater. Infant. Recife, v. 5, n. 3, p. 337-348, set. 2005.

BRASIL. Ministério da Saúde. Caderno de Atenção Básica: Saúde Mental. Situações de saúde mental comuns na Atenção Básica. Brasília, 2003. Disponível em: <http://189.28.128.100/dab/docs/portaldab/publicacoes/caderno_34.pdf>. Acesso em:12 de junho de 2018.

BRASIL. Ministério da Saúde. Saúde Mental em Dados - 12, ano 10, no 12. Informativo eletrônico. Brasília: outubro de 2015. Disponível em: http://www.mhinnovation.net/sites/default/files/downloads/innovation/reports/Report_12edicao-do-Saude-Mental-em-Dados.pdf. Acesso em 16 de junho de 2018.

CAMBOTA, Jacqueline Nogueira; ROCHA, Fabiana Fontes. Determinantes das desigualdades na utilização de serviços de saúde: análise para o Brasil e regiões. 2015.

CARDOSO, Lucilene; FRARI GALERA, Sueli Aparecida. Doentes mentais e seu perfil de adesão ao tratamento psicofarmacológico. Revista da Escola de Enfermagem da USP. V. 43, n. 1, 2009.

CARVALHO, Marcos Danúbio Alves de et al. Perfil epidemiológico dos usuários da rede de saúde mental do município de Iguatu, CE. SMAD. Revista eletrônica saúde mental álcool e drogas. V. 6, n. 2, p. 337-349, 2010.

CARLOTTO, Mary Sandra. Transtornos Mentais Comuns em trabalhadores de Unidades Básicas de Saúde: Prevalência e fatores associados. Psicologia Argumento. V. 34, n. 85, 2017.

CRUZ, Daniela Cordeiro et al. Perfil de Pacientes com Transtornos Mentais atendidos no Centro de Atenção Psicossocial do Município de Candeias-Bahia. Revista Brasileira de Ciências da Saúde. V. 20, n. 2, p. 93-98, 2016. 
FIOROTTI, Cecilia; TOMAZELLI, Jeane; MALAGRIS, Lucia. Transtornos mentais comuns em pacientes hipertensos: estudo em unidade de atenção primária à saúde no Rio de Janeiro. Revista de APS. v. 12, n. 3, 2009.

FREITAS, Aline Araújo; SOUZA, Rozemere Cardoso de. Caracterização clínica e sociodemográfica dos usuários de um Centro de Atenção Psicossocial (CAPS). Revista Baiana de Saúde Pública. v. 34, n. 3, p. 530-543, 2010.

IBGE. Instituto Brasileiro de Geografia e Estatística. Pesquisa Nacional de Saúde 2013: percepção do estado de saúde, estilos de vida e doenças crônicas: Brasil, grandes regiões e unidades da federação. 2014.

JOEL, Daphna et al. Sex beyond the genitalia: The human brain mosaic. Proceedings of the National Academy of Sciences of the United States of America. V. 112, n. 50, p. 15468-15473, nov. 2015.

MANGUALDE, Alice Ananias et al. Perfil epidemiológico dos pacientes atendidos em um Centro de Atenção Psicossocial. Mental. v. 10, n. 19, 2013.

OLIVEIRA, Valdimeire Ferreira et al. Caracterização de pacientes com transtornos mentais atendidos no centro de atenção psicossocial em São Francisco do Conde-Bahia. Revista de Ciências Médicas e Biológicas. V. 13, n. 2, p. 204-211, 2015.

PATEL, Vikram; KLEINMAN, Arthur. Poverty and common mental disorders in developing countries. Bulletin of the World Health Organization. V. 81, p. 609-615, 2003.

PELISOLI, Cátula da Luz; MOREIRA, Ângela Kunzler. Caracterização epidemiológica dos usuários do Centro de Atenção Psicossocial Casa Aberta. Rev. psiquiatr. Rio Gd. Sul. V. 27, n. 3, p. 270-277, 2005.

PEREIRA, Maria Odete et al. Perfil dos usuários de serviços de Saúde Mental do município de Lorena-São Paulo. Acta Paulista de Enfermagem. V. 25, n. 1, p. 48-54, 2012.

REIS, Leonardo Naves dos; REISDORFER, Emilene; GHERARDI-DONATO, Edilaine Cristina da Silva. Perfil dos usuários com diagnóstico de transtornos de personalidade de um serviço de saúde mental. SMAD. Revista eletrônica saúde mental álcool e drogas. V. 9, n. 2, p. 70-75, 2013.

WHO. World Health Organization. Mental health action plan 2013-2020. World Health Organization, 2013. Disponível em: < https://apps.who.int/iris/bitstream/handle/10665/89966/9789241506021_eng.pdf?sequenc $\mathrm{e}=1 \mathrm{l}$. . Acesso em: 8 de Julho de 2019 . 